\title{
1
}

\section{The ABCs of LGBTQ}

Many terms and letters have been used to describe the LGBTQ communities. Outsiders looking in may feel that the letters are overwhelming, academic, or inaccessible to the rest of the population. The intention of these letters is to give identity to a group that was often just called "the gay community." Although the attempt of the acronym was to be inclusive, the lettering can also be limiting. For the purposes of this book, the acronym LGBTQ is used, but it is by no means a definitive descriptor of the population.

In this chapter, you will learn:

1. The definition of LGBTQ terms and definitions

2. How to use these common terms

\section{LGBTQ EXPLAINED}

The language that is used to talk about LGBTQ people continues to evolve and expand. "LGBT" became popular in the late 20th century. The addition and use of the "Q" gained traction as the 20th century turned into the 21st. Many variations of the acronym exist with some gaining popularity in various different settings. It may seem that while new terms are appearing, forgotten or unused terms are becoming mainstream and words once viewed as derogatory are being reclaimed and commonplace. 
For nurses, it is important to understand the basics of the language that LGBTQ people use to identify themselves. An example of this evolution is the use of the word "queer." Once a pejorative, many people have reclaimed this word to describe their sexual or gender identity. Understand that although one patient may describe themselves as queer, this term could potentially be divisive to some patients because of its history. LGBTQ people today have a large vocabulary with which they can articulate their gender identity and their sexual orientations. These words can make some nurses and healthcare providers feel uncomfortable.

\section{Fast Facts}

By asking, learning, and understanding terminology, abbreviations, and acronyms association with LGBTQ people can help nurses to confront their biases or assumptions and promote culturally competent care.

In nursing and healthcare, it might be common to see the LGBTQ community called sexual and gender minorities. Researchers may use sexual orientation and gender identity (SOGI) when referring to data about LGBTQ people. In public health, men who have sex with men (MSM) and women who have sex with women (WSW) may also be used to understand a person's sexual habits without referring to their sexual orientation. Understanding these acronyms and terms can help nurses to understand their patients, become an ally to LGBTQ people, and allow us to facilitate conversations with our patients (see Box 1.1). Most importantly, it is the first step in listening to who patients are as people.

\section{LGBTQ TERMS AND DEFINITIONS}

Affirmed gender (noun): The gender by which one wishes to be known. This term is often used to replace terms like "new gender" or "chosen gender," which imply that a person's gender was chosen rather than simply innate.

Agender (adj.): Describes a person who does not identify with any gender identity.

Ally (noun): A person who does not identify as LGBTQ but stands with and advocates for LGBTQ people.

Androgynous (adj.), Androgyne (noun): Used to describe someone who identifies or presents as neither distinguishably masculine nor feminine. 


\section{BOX 1.1 LGBTQ DEFINED}

- L(Lesbian): A lesbian is a woman who feels a sexual and romantic attraction to other women.

- G (Gay): Gay is usually a term used to refer to men who feel sexual and romantic attraction to other men. Lesbians can also be referred to as gay.

- B (Bisexual): Bisexual indicates having a romantic and sexual attraction to both men and women.

- $\mathbf{T}$ (Transgender): Transgender is a term that indicates that a person's gender identity or expression is different from the sex they were assigned at birth.

- Q (Queer or Questioning): This initial usually represents queer or questioning. Queer is considered an umbrella term for anyone who is non-cisgender or heterosexual. Queer may be used by people who feel that another term such as gay, lesbian, or bisexual is too limiting or not representative of their identity. Questioning refers to people who may be unsure of their sexual orientation or gender identity.

Other variations:

- LGBTQIA: This acronym includes initials for queer, intersex, and asexual.

- LGBTIQA+: This variation stands for lesbian, gay, bisexual, transgender, intersex, queer/questioning, asexual, and others that can include pansexual and nonbinary.

Sources: GLAAD \& HRC.

Aromantic ( $a d j.):$ A romantic orientation generally characterized by not feeling romantic attraction or a desire for romance.

Asexual (adj.): Used to describe people who do not experience sexual attraction or do not have a desire for sex. Many experience romantic or emotional attractions across the entire spectrum of sexual orientations. Asexuality differs from celibacy, which refers to abstaining from sex. Also ace or ace community.

Assigned sex (noun): The sex that is assigned to an infant at birth based on the child's visible sex organs, including genitalia and other physical characteristics. Often corresponds with a child's assigned gender and assumed gender.

Binary system (noun): Something that contains two opposing parts; binary systems are often assumed despite the existence of a 
spectrum of possibilities. Gender (man/woman) and sex (male/ female) are examples of binary systems often perpetuated by our culture.

Biological sex (noun): A medical classification that refers to anatomical, physiological, genetic, or physical attributes that determine if a person is assigned male, female, or intersex identity at birth. Biological sex is often confused or interchanged with the term "gender," which encompasses personal identity and social factors, and is not necessarily determined by biological sex. See gender.

Cisgender (adj.): Describes a person whose gender identity (defined later in this section) aligns with the sex assigned to them at birth.

Cissexism (noun): A system of discrimination and exclusion that oppresses people whose gender and/or gender expression falls outside of normative social constructs. This system is founded on the belief that there are, and should be, only two genders-usually tied to assigned sex.

Coming out (verb): A lifelong process of self-acceptance and revealing one's queer identity to others. This may involve something as private as telling a single confidant or something as public as posting to social media.

Demisexual (adj.): Used to describe someone who feels sexual attraction only to people with whom they have an emotional bondoften considered to be on the asexual spectrum.

Gender (noun): A set of social, physical, psychological, and emotional traits, often influenced by societal expectations, that classify an individual as feminine, masculine, androgynous, or other. Words and qualities ascribed to these traits vary across cultures.

Gender dysphoria (noun): Clinically significant distress caused when a person's assigned birth gender is not the same as the one with which they identify.

Gender expression (noun): External appearance of one's gender identity, usually expressed through behavior, clothing, haircut, or voice, which may or may not conform to socially defined behaviors and characteristics typically associated with being masculine or feminine.

Gender-fluid ( $a d j$.): A person who does not identify with a single fixed gender and whose identification and presentation may shift, whether within or outside of the male/female binary.

Gender identity (noun): One's innermost feeling of maleness, femaleness, a blend of both, or neither. One's gender identity can be the same or different from their sex assigned at birth.

Gender-neutral (adj.): Not gendered, usually operating outside the male/female binary. Can refer to language (e.g., pronouns), spaces (e.g., bathrooms), or identities. 
Gender nonconforming (adj.): A broad term referring to people who do not behave in a way that conforms to the traditional expectations of their gender or whose gender expression does not fit neatly into a category.

Genderqueer ( $a d j$.$) : Describes a person who rejects static catego-$ ries of gender (i.e., the gender binary of male/female) and whose gender expression or identity falls outside of the dominant social norms of their assigned sex. They may identify as having aspects of both male and female identities or neither.

Gender roles (noun): The social behaviors and expression that a culture expects from people based on their assigned sex (e.g., girls wear pink; boys do not cry; women care for home and child; men are more violent), despite a spectrum of various other possibilities.

Heteronormativity (noun): Coined by social critic Michael Warner, the term refers to a societal assumption of certain norms:

1. There are two distinct sexes.

2. Male and female functions and characteristics are distinctly different.

3. Traits such as attraction and sexual behavior correspond to anatomy.

Those who do not fit these norms-be it through same-sex attraction, a nonbinary gender identity, or nontraditional gender expression-are therefore seen as abnormal and often marginalized or pressured to conform to norms as a result.

Heterosexism (noun): The assumption that sexuality between people of different sexes is normal, standard, superior, or universal while other sexual orientations are substandard, inferior, abnormal, marginal, or invalid.

Heterosexual (adj.): Used to describe people whose enduring physical, romantic, and/or emotional attraction is to people of the opposite sex. Also straight.

Heterosexual/cisgender privilege (noun): Refers to societal advantages that heterosexual people and cisgender people have solely because of their dominant identities. This can include things as simple as safely holding hands with a romantic partner in public or having safe access to public bathrooms. This can also include systemic privileges such as the right to legally donate blood, to adopt children without facing possible rejection because of your sexual orientation, or to play organized sports with others of the same gender identity.

Homophobia (noun): A fear or hostility toward lesbian, gay, and/ or bisexual people, often expressed as discrimination, harassment, and violence. 
Intersex (adj.): An umbrella term describing people born with reproductive or sexual anatomy and/or a chromosome pattern that cannot be classified as typically male or female.

Latinx (adj.): A gender-expansive term for people of Latin American descent used to be more inclusive of all genders than the binary terms Latino or Latina.

Misgender (verb): To refer to someone in a way that does not correctly reflect the gender with which they identify, such as refusing to use a person's pronouns or name.

Nonbinary (adj.): An umbrella term that refers to individuals who identify as neither man or woman, or as a combination of man or woman. Instead, nonbinary people exhibit a boundless range of identities that can exist beyond a spectrum between male and female.

Outing (verb): The inappropriate act of publicly declaring (sometimes based on rumor and/or speculation) or revealing another person's sexual orientation or gender identity without that person's consent.

Pansexual (adj.): Used to describe people who have the potential for emotional, romantic, or sexual attraction to people of any gender identity, though not necessarily simultaneously, in the same way, or to the same degree. The term panromantic may refer to a person who feels these emotional and romantic attractions but identifies as asexual.

Preferred pronouns (adj.): The pronoun or set of pronouns that an individual personally uses and would like others to use when talking to or about that individual. Can include variations of he/him/his, she/her/hers, and they/their/theirs, among others. This term is being used less and less in LGBTQ circles, as it suggests one's gender identity is a "preference" rather than innate. Recommended replacement: "Your pronouns, my pronouns, their pronouns, and so forth."

Queer (adj.): Once a pejorative term, a term reclaimed and used by some within academic circles and the LGBTQ community to describe sexual orientations and gender identities that are not exclusively heterosexual or cisgender.

Questioning (adj.): A term used to describe people who are in the process of exploring their sexual orientation or gender identity.

Same-gender loving (adj.): This term was and is used by some members of the Black community who feel that terms like gay, lesbian, and bisexual (and sometimes the communities therein) are Eurocentric and fail to affirm Black culture, history, and identity.

Sexual orientation (noun): An inherent or immutable emotional, romantic, or sexual attraction to other people; oftentimes used to 
signify the gender identity (or identities) to which a person is most attracted.

Third gender (noun): A gender identity that is neither male nor female, existing outside the idea that gender represents a linear spectrum between the two. Sometimes a catchall term or category in societies, states, or countries that legally recognize genders other than male and female.

Transgender (adj.): An umbrella term for people whose gender identity differs from the sex they were assigned at birth. Not all trans people undergo transition. Being transgender does not imply any specific sexual orientation. Therefore, transgender people may identify as straight, gay, lesbian, bisexual, or something else. Also, trans.

Transitioning (verb): A process during which some people strive to more closely align their gender identity with their gender expression. This includes socially transitioning, during which a person may change their pronouns, the name they ask to be called, or the way they dress to be socially recognized as another gender. This includes legal transitioning, which may involve an official name change and modified IDs and birth certificates. And this includes physically transitioning, during which a person may undergo medical interventions to more closely align their body to their gender identity. Transgender and nonbinary people transition in various ways to various degrees; self-identification alone is enough to validate gender identity.

Transphobia (noun): The fear and hatred of, or discomfort with, transgender people. This may manifest into transphobic actions, such as violence, harassment, misrepresentation, or exclusion.

Transsexual (adj.): A less frequently used term (considered by some to be outdated or offensive), which refers to people who use medical interventions such as hormone therapy, gender-affirming surgery (GAS), or sex reassignment surgery (SRS) as part of the process of expressing their gender. Some people who identify as transsexual do not identify as transgender and vice versa. Only use this term if someone who specifically identifies as such asks you to.

Two spirit (adj.): An umbrella term in Native culture to describe people who have both a male and female spirit within them. This encompasses many tribe-specific names, roles, and traditions, such as the winkte of the Lakota and nadleeh of the Navajo people. This term often describes Native people who performed roles and gender expression associated with both men and women. This term should be used only in the context of Native culture. 


\section{Fast Facts}

Words are important to LGBTQ people as they can provide a person with a sense of identity and a way to describe themselves. However, they can also be limiting and not capture the full identity of the diverse population that is the LGBTQ population. Nurses should have a basic understanding of the various terms and vocabulary surrounding LGBTQ people so they are able to better understand and listen to their patients.

\section{Further Reading}

GLAAD Media Reference Guide-Lesbian/gay/bisexual glossary of terms. (2016, October 26). Retrieved January 15, 2021, from https://www.glaad .org/reference/lgbtq

Gold, M. (2018, June 21). The ABCs of L.G.B.T.Q.I.A.+. Retrieved January 15, 2021, from https://www.nytimes.com/2018/06/21/style/lgbtq-gender -language.html

Human Rights Campaign. (n.d.). Glossary of terms. Retrieved January 15, 2021, from https://www.hrc.org/resources/glossary-of-terms

$L G B T Q+$ glossary. (n.d.). Retrieved January 15, 2021, from https://health .ucdavis.edu/diversity-inclusion/LGBTQI/LGBTQ-Plus.html

Mayer, K. H., Potter, J., Goldhammer, H., \& Makadon, H. J. (2015). The Fenway guide to lesbian, gay, bisexual, and transgender health. American College of Physicians. 